\title{
Clinical Characteristics, Maternal and Neonatal Outcomes of COVID-19 Positive Pregnant Mothers at a Tertiary Hospital in Johannesburg, South Africa
}

Tanusha Devi Ramdin ( $\nabla$ tanusha.ramdin@wits.ac.za )

Witwatersrand Health Sciences: University of the Witwatersrand Faculty of Health Sciences https://orcid.org/0000-0002-9490-6916

Rossella Marina Bandini

Wits University: University of the Witwatersrand

Robin Terance Saggers

Witwatersrand Health Sciences: University of the Witwatersrand Faculty of Health Sciences

Michael Radomsky

Witwatersrand Health Sciences: University of the Witwatersrand Faculty of Health Sciences

Mphelekedzeni Mulaudzi

Witwatersrand Health Sciences: University of the Witwatersrand Faculty of Health Sciences

Shastra Aventra Bhoora

Witwatersrand Health Sciences: University of the Witwatersrand Faculty of Health Sciences

Daynia Elizabeth Ballot

Witwatersrand Health Sciences: University of the Witwatersrand Faculty of Health Sciences

\section{Research article}

Keywords: COVID-19, maternal, neonatal, low-middle income countries, South Africa

Posted Date: August 9th, 2021

DOI: https://doi.org/10.21203/rs.3.rs-751147/v1

License: (a) (1) This work is licensed under a Creative Commons Attribution 4.0 International License. Read Full License

Version of Record: A version of this preprint was published at Journal of Pediatrics, Perinatology and Child Health on January 1st, 2021. See the published version at https://doi.org/10.26502/jppch.74050085. 


\section{Abstract}

Background: The global spread of the novel coronavirus 2019 (COVID-19) has been sudden shock to the world and resulted in many questions that remain unanswered. Limited data has been published in lowmiddle-income country settings (LMICS)

Objectives: To describe the clinical characteristics and outcome of mothers and neonates delivered from COVID-19 positive mothers, and to identify the incidence of COVID-19 positive neonates.

Methods: A prospective, descriptive study, from 1 August 2020 to 31 March 2021 conducted at a tertiary hospital, in Johannesburg

All neonates born to mothers that were COVID-19 positive and that required admission to the neonatal unit were included. Informed consent was obtained from mothers prior to enrolment.

Results: A total of 111 COVID-19 positive pregnant women delivered neonates at the tertiary hospital. In this study, only 28 of the 111 (25\%) neonates born to COVID-19 positive mothers were admitted. The majority of the COVID-19 pregnant mothers were asymptomatic or had mild symptomatic disease (80\%). Two $(2 / 111(2 \%)$ mothers required ICU admission and three $(3 / 111(3 \%)$ of them demised.

In relation to neonatal outcomes, the majority of the neonates were delivered at a gestational age of 35 weeks with a birth weight of 2400 grams. The most common symptom was respiratory distress (89\%).

The one (3\%) neonate that tested positive for COVID-19 was born moderately preterm with a low birth weight and respiratory distress syndrome. Two $(2 / 28(7 \%)$ neonates demised, however the cause of death was not related to COVID-19. All the remaining (26/28 (93\%) neonates were discharged and were well on follow up.

Conclusion: Our study has shown that the risk of neonatal transmission from pregnant COVID-19 mothers is relatively low, and the majority of neonatal disease ranged from asymptomatic to mildly symptomatic disease. Further research efforts are essential to improve neonatal care in LMICS.

\section{Background}

In December 2019, several pneumonia cases of unknown origin emerged in Wuhan, Hubei province, in the Republic of China. On 11 March 2020, the World Health Organization (WHO) labelled the disease associated with the novel coronavirus 2019 (COVID-19) a pandemic.[1] The global spread was sudden, and many questions remain unanswered.

The virus is highly contagious via direct contact and droplet spread with a mortality rate of $3.4 \%$ according to the WHO.[1] As of 27 April 2021, South Africa (SA) had 1576320 positive cases of COVID19 resulting in 54186 deaths.[2] Recently two mutated variants have been identified in SA, 501Y.V2 (or B.1.351) and 501Y.V1 or (B.1.1.7) respectively. The new variants may help the virus spread more easily 
and affect the antibody response. The government implemented a strict lockdown of the country to help curb the spread of the virus in March 2020. Since then, the lockdown has been eased but the country remains in varying degrees of lockdown.

South Africa is a low-middle income country (LMICS) with very dense informal settlements areas that may represent a challenge when considering the transmissibility of COVID-19.[3] Of concern is the huge local burden of other diseases like tuberculosis (TB) and Human Immunodeficiency Virus (HIV). The WHO was initially hopeful that those who have achieved viral suppression through highly active antiretroviral therapy (HAART) may show less severe symptoms of COVID-19. In SA, only $63 \%$ of HIV positive population are on HAART.[4] The South African maternal population is very different to Asia, Europe or the United States (US). Approximately $28.6 \%$ of our mothers are HIV positive and only about half of the mothers are on HAART.[5] TB is also prevalent in pregnant women.[5, 6] It was also found that Bacille Calmette-Guerin (BCG) childhood vaccination might offer broad protection to respiratory infections and it may reduce the number of COVID-19 cases in the country. [7]

The clinical presentation of COVID-19 in adults commonly includes fever, cough, fatigue, shortness of breath, and rarely diarrhoea.[8] At time of diagnosis approximately $80 \%$ of cases are mild or moderate in adults, $15 \%$ of cases are severe requiring hospital admission and $5 \%$ of cases are critical requiring intensive care.[8] Studies have shown that children tend to have milder disease than adults.[8, 9] Severe acute respiratory syndrome coronavirus 2 (SARS-CoV-2)(the virus causing COVID-19) is diagnosed from genetic material ribonucleic acid (RNA) in samples from the respiratory tract.

Of increasing concern is the pregnant women population that are more susceptible to COVID-19 and are likely to have complications.[10-13]

The unanswered questions:

1. Whether pregnant women are more severely affected or may be asymptomatic than the general population? Whether intrauterine or perinatal transmission occurs is largely unknown?

2. Whether SAR-CoV-2 is transmitted in breast milk. Limited information has suggested that SARS-COV2 is not transmitted through breast milk but the numbers on which to determine this evidence is small?

3. Lastly, postnatal transmission from an infected mother to a well new-born. This is controversial as it raises the question of temporary separation of infected mothers and their neonates?

Several case reports have looked at outcomes of pregnant mothers with COVID-19 and their neonates. [13-16] Reassuringly, mothers and their babies generally appear to do well.[17, 18] Strong evidence suggests amniotic fluid, placental swabs and cord bloods have consistently tested negative for SARSCoV-2.[12, 14, 19-21] However, further investigation is required to determine if COVID-19 may damage the placenta.[20] A few cases of neonates delivered to mothers with COVID-19 had increased SARS-CoV-2 immunoglobulin $\mathrm{M}(\mathrm{IgM})$ levels. $[14,19,20]$ This may suggest intrauterine transmission, however reverse transcription polymerase chain reaction (RT-PCR) for SARS-CoV-2 from these neonates was negative, the 
IgM may be a false positive.[19] It is not known if the developing foetus is exposed to a community of viruses (virome).[22] Looking more closely at the virome in the amniotic fluid may detect COVID-19.

The question of vertical transmission is especially relevant given the recent history of vertical maternalfetal transmission of such emerging viral infections as the Zika virus, Ebola virus, Marburg virus and other agents which can threaten the health and survival of an infected mother and foetus [22]. In many studies, most neonates have not acquired COVID-19.[23] Information from nine Chinese mothers revealed there was no evidence of vertical transmission of COVID-19 during pregnancy.[12] The impact of COVID19 in pregnancy still remains unclear but in addition to the potential for vertical transmission the disease may lead to neonatal complications such as preterm birth as a consequence of maternal fever.

All the breast milk samples from these mothers after the first lactation tested negative for SARS-CoV-2. In LMICS breastfeeding has been traditionally a protective factor against diarrhoea and respiratory infections and death.[24] The benefit of breastfeeding especially with no cases of COVID-19 being transmissible through it still outweighs risks associated with formula feeding. Alternatives to breast feeding are expressed breast milk or donor breast milk.[25] The mothers should adhere to strict hand hygiene, wear a mask, and routinely clean and disinfect surfaces during breastfeeding to reduce the risk of transmission.

There is uncertainty if a well-baby should be isolated from COVID-19 positive mothers.[26, 27] In LMICS there are not enough isolation areas to cohort well babies. The WHO advocates that mothers should not be separated from their neonates, unless medically indicated.[26] This will also minimize the disruption to breastfeeding during stay in facilities providing maternity and neonate services

The definition for neonates suspected to have COVID-19 infection is those neonates born to mothers with a history of COVID-19 infection in the 14 days prior to delivery and/or 28 days after delivery, or the neonate is directly exposed to those infected with COVID-19 (including family members, caregivers, medical staff and visitors).[28] The diagnosis of COVID-19 neonatal infection should meet the following criteria $[28,29]$ :

1. At least one clinical symptom, including unstable body temperature, low activity or poor feeding or shortness of breath.

2. Chest radiographs showing abnormalities, including unilateral or bilateral milled glass opacities.

3. A COVID-19 diagnosis in patient's family or caregiver

4. A close contact with people who may have or have confirmed COVID-19, or patients with unexplained pneumonia.

Clinical findings, especially in premature infants, are not specific. Therefore, it is necessary to closely monitor vital signs, respiratory symptoms, and gastrointestinal symptoms. Laboratory findings may be non-specific. COVID-19 can be detected in samples taken from the upper respiratory tract (nasopharyngeal and oropharyngeal), the lower respiratory tract (endotracheal aspirate, bronchoalveolar 
lavage), blood and stool. Currently, the principles of treatment are supportive care and treatment of complications.

This study aimed to identify the incidence of neonatal COVID-19 within our unit, and to describe the clinical characteristics and outcome of mothers and neonates delivered to COVID-19 positive mothers.

\section{Methods}

\section{Study design, period, and setting:}

This was a prospective, single-centre, descriptive study. The study took place at a tertiary hospital, Charlotte Maxeke Johannesburg Academic Hospital (CMJAH), in Johannesburg, South Africa. CMJAH has a 100-bed neonatal unit and a combined 15 bed neonatal and paediatric intensive care unit. The recruitment period was eight months from 1 August 2020 to 31 March 2021.

\section{Study population:}

Pregnant women at CMJAH who were diagnosed with COVID-19 were eligible for inclusion. Pregnant women that tested positive for COVID-19 during their first and second trimester of pregnancy were excluded. COVID-19 was diagnosed with a positive SARS-CoV-2 PCR test. All neonates born to mothers who were COVID-19 positive were included. Stillbirths and neonates with missing records were excluded.

\section{Procedures:}

Well neonates were housed with their mothers and sick neonates were transferred to a designated neonatal COVID-19 isolation area in the neonatal unit. Patients were treated according to standard of care and unit policy. Maternal and neonatal epidemiology, demographics, clinical signs and symptoms, laboratory results, delivery method, treatments and follow up visits were captured on the Research Electronic Data Capture (REDCap ${ }^{\mathrm{TM}}$ ) database,[31] which is hosted by the University of the Witwatersrand.

Nasopharyngeal or oropharyngeal specimens were obtained from mother and neonate at admission in a viral transport medium. Laboratory confirmation for SARS-CoV-2 infection were performed by the National Health Laboratory Services (NHLS) and confirmed by real time polymerase chain reaction (RTPCR). The mode of delivery was at the discretion of the attending obstetrician.

\section{Statistical analysis:}

Statistical analysis was performed using SPSS, version 27 (IBM, USA). Continuous variables were described using measures of central tendency - mean and standard deviation for those variables with a normal distribution and median and range for those variables with a skewed distribution. Categorical variables were described using frequencies and percentages.

\section{Results}




\section{Sample size}

The median maternal age was 31 years and the mean gestational age of neonates was 35.5 weeks. The mean birth weight was 2400 grams. The median Apgar score at 5 minutes was 9 .

Twenty-eight neonates born to positive COVID-19 mother were admitted to the neonatal unit. Among symptomatic neonates the most common clinical presentation was respiratory distress $(89.29 \%)$ and feeding intolerance $(42.86 \%)$. 
Table 1

Characteristics of mothers that tested positive for COVID-19 at a tertiary hospital in Johannesburg, South Africa, 1 August 2020 to 31 March 2021.

\begin{tabular}{ll}
\hline Variable & Frequency \\
\hline Maternal comorbidity & $11(39.3)$ \\
\hline HIV & $7(25.0)$ \\
\hline TB & $2(7.1)$ \\
\hline PET and its complications & \\
\hline Mode of delivery & $22(78.6)$ \\
\hline Caesarean section & $6(21.4)$ \\
\hline Normal Vaginal Delivery & 0 \\
\hline Contact with COVID-19 positive person & 0 \\
\hline Travel outside the country & \\
\hline Maternal COVID-19 relevant symptoms & $9(32.1)$ \\
\hline Fever (>37.5C) & $9(32.1)$ \\
\hline Cough & $2(7.1)$ \\
\hline Dyspnoea & 0 \\
\hline Diarrhoea & $27(96.4)$ \\
\hline Mothers condition & $1(3.6)$ \\
\hline Asymptomatic & $3(10.7)$ \\
\hline Mild & $19(67.9)$ \\
\hline Moderate & $5(17.9)$ \\
\hline Severe (ICU admission) & $1(3.6)$ \\
\hline Maternal Outcome & \\
\hline Survived & \\
\hline Demised & \\
\hline
\end{tabular}

HIV = Human Immunodeficiency Virus; TB = tuberculosis; $P E T$ = pre-eclampsia; ICU = intensive care unit. 
Table 2

Characteristics of neonates born to positive COVID-19 mothers admitted to the neonatal unit in a tertiary hospital in Johannesburg,

South Africa, 1 August 2020-31 March 2021.

\begin{tabular}{|c|c|}
\hline Variable & Frequency $\mathbf{N}(\%)$ \\
\hline \multicolumn{2}{|c|}{ Need for resuscitation in the delivery room } \\
\hline Nasal prong oxygen & $24(85.7)$ \\
\hline Bag mask ventilation & $14(50.0)$ \\
\hline Intubation & $4(14.3)$ \\
\hline \multicolumn{2}{|c|}{ COVID-19 PCR swab result } \\
\hline Positive & $1(3.6)$ \\
\hline Negative & $27(96.4)$ \\
\hline \multicolumn{2}{|l|}{ Neonatal complications } \\
\hline Fever $\left(>37.5^{\circ} \mathrm{C}\right)$ & $3(10.7)$ \\
\hline Respiratory Distress & $25(89.3)$ \\
\hline Feeding Abnormalities & $12(42.9)$ \\
\hline Asymptomatic & $1(3.6)$ \\
\hline \multicolumn{2}{|l|}{ Neonatal Outcome } \\
\hline Survived & $26(92.9)$ \\
\hline Demised & $2(7.1)$ \\
\hline
\end{tabular}


Table 3

Blood results and management of neonates born to COVID-19 positive mothers at a tertiary hospital in Johannesburg, South Africa, 1 August 2020 to 31 March 2021.

\begin{tabular}{|ll|}
\hline Parameter & Mean (SD) \\
\hline White Cell Count $\left(\times 10^{9} / \mathrm{L}\right)$ & 11.87 \\
\hline Haemoglobin $(\mathrm{g} / \mathrm{dL})$ & 15.87 \\
\hline Platelets $\left(\times 10^{9} / \mathrm{L}\right)$ & 256.48 \\
\hline Elevated CRP on day 2 $(>10)$ & $5(17.86)$ \\
\hline Treatment & $\mathbf{N}(\%)$ \\
\hline Empiric antibiotics & 27 \\
\hline Targeted antibiotics & 1 \\
\hline Surfactant and nCPAP & 8 \\
\hline CRP = C-reactive protein; nCPAP = nasal continuous positive airway pressure \\
\hline
\end{tabular}

\section{Discussion}

The COVID-19 pandemic has caused severe disease in adults, including pregnant women, while rarely in neonates.[32] Critical gaps in the literature exist, and important research is still required. In LMICS there is a paucity of information on COVID-19 in neonates and their outcomes. Our study reports the maternal characteristics and neonatal outcomes of neonates born to COVID-19 positive mothers.

Rates of COVID-19 infection in pregnant women vary geographically and could potentially reflect the prevalence of the virus in the general population. In August 2020, universal screening for COVID-19 was adopted for all pregnant women at our centre. The benefits of a universal testing approach include the ability to use COVID-19 status to determine hospital isolation practices and bed assignments, inform neonatal care, and guide the use of personal protective equipment.(32) Data from a New York City study of 215 pregnant women that were screened for COVID-19, found 33 to be positive for COVID-19, of whom 29 (87.9\%) were asymptomatic.[33] Our study supports the findings of other studies that showed that majority of mothers that tested positive for COVID-19 were asymptomatic or had mild symptoms.[17, 34 , 35]

Several studies have shown foetal complications of maternal COVID-19, including prematurity and stillbirth. $[13,36]$ In our study there was a higher occurrence of prematurity (mean gestational age was 35.5 weeks) and low birth weight infants (mean birth weight was 2400 grams). This is similar to reports from high income country setting (HICS) and LMICS.[13, 32] A possible explanation for this is the evidence of hypercoagulability in pregnant women with COVID-19 disease.[32] Due to this, it has been hypothesised there is decreased perfusion to maternal/foetus and placenta vasculature, and this would 
result in intrauterine growth restriction and more so increase preterm deliveries. More rigorous studies are needed to clarify the impact of COVID-19 on the physiology of pregnancy and foetus.

Risk factors for maternal transmission of the disease to the neonate are not fully understood. It remains unclear if severity of maternal disease, time of acquisition, gestational age at delivery, or mode of delivery contributes to infection and transmission. Our unit is a tertiary facility with referrals of all complicated pregnancies in the surrounding area, therefore the majority of deliveries were by caesarean section (78.6\%), but mode of delivery was at the discretion of the obstetrician.

Perinatal transmission of COVID-19 may occur via transplacental route or through respiratory droplets after birth. Some reports of positive anti SARS-CoV-2 immunoglobulin M (IgM) and immunoglobulin G $(\mathrm{IgG})$ in the neonate or placental histopathological findings were inconclusive.[14, 23] Thus far, several studies have shown low rates of perinatal acquisition among neonates born to mothers with COVID-19 in the United States, China, Italy, Sweden and the United Kingdom. $[15,17,34]$ Our results agree with findings of other studies, in which mother to neonate vertical transmission were low. Only one neonate tested positive for COVID-19 (3.6\%). The possibility of transmission via breastmilk is unclear.[37] Therefore, our centre strongly advocates breastfeeding despite mothers being positive for COVID-19. We advise strict infection control methods between neonate and mother, including hand washing and maternal maskwearing. The benefit of breastmilk far outweighs the unknown risk possible transmission, especially in LMICS.

The majority of neonates born to COVID-19 positive mothers had mild disease, however some studies clinical presentation varies from asymptomatic carriage to critical illness.[15, 17, 32] Many hypotheses have been suggested to explain this fact, including a lower ACE2 expression, the receptor that SARS-CoV2 uses for host entry, less proinflammatory cytokine response, a stronger innate immune response and a higher proportion of total lymphocytes and absolute numbers of $T$ and $B$ cells.[18]

Only one (3.6\%) neonate tested positive for COVID-19. Laboratory investigations were normal. The COVID19 positive neonate had mild disease. These results mirror those of previous studies that have examined clinical presentation of neonates born to COVID-19 positive mothers.[34, 38] The two $(7.1 \%)$ neonates that demised were COVID-19 PCR negative and the deaths were unrelated to COVID-19: one neonate died from an inoperable cardiac disease and the other from severe immaturity and nosocomial sepsis. However, it is difficult to distinguish respiratory distress syndrome from COVID-19 since the chest radiograph may have similar features. Neonates are considered a vulnerable population as limited studies have shown neonates to be at risk of experiencing severe disease compared to older children.[34]

\section{Conclusion}

Our study showed the risk of neonatal transmission to be low. Most of the neonatal disease ranged from asymptomatic to mildly symptomatic disease. However, COVID-19 has created unparalleled challenges for the global healthcare system. Many knowledge gaps remain including maternal to neonatal 
transmission and clinical presentations of pregnant women and neonates as well as long-term neurodevelopmental outcome. Further research efforts are essential to improve neonatal care.

\section{Limitations}

This study was limited by it being a single centre study with a small sample size. The study only included neonates born to COVID-19 positive mothers and admitted to the neonatal unit.

\section{Abbreviations}

COVID-19 - coronavirus 19

Bacille Calmetie-Gurein - BCG

CMJAH - Charlotte Maxeke Johannesburg Academic Hospital

CRP - C Reactive Protein

ICU - Intensive care unit

IgG - Immunoglobulin G

IgM - Immunoglobulin M

HAART- highly active antiretroviral therapy

HIV- Human Immunodeficiency virus

HICS- high income country settings

LMICS - low middle income country settings

nCPAP - nasal continuous airway pressure

NHLS - National Health Laboratory service

REDCap - Research Electronic Data Capture

RT-PCR - Real time polymerase chain reaction

RNA - ribonucleic acid

PET - pre-eclampsia

SARS-CoV2 - severe acute respiratory syndrome coronavirus 2 
TB - Tuberculosis

WHO - World Health Organization

US - United States

Declarations

\section{Ethics approval and consent to participate}

Ethics was approved by Witwatersrand University Human Rsearch Ethics Committee (M200534). Mothers signed informed consent prior to enrollment of mother and neonate in the study.

\section{Consent of publication}

Not applicable.

\section{Availability of data and material}

Data and material are available on request.

\section{Competing Interest}

None.

\section{Funding}

A Self-Initiated Research Grant from the South African Medical Research Council funded this study (DEB). The grant assisted with travel costs for parents and participants to follow up clinic visits.

\section{Author Contributions}

TR conceptualized the study, performed data collection, analysis and prepared the various drafts for publication. MR and SB assisted with the data collection. RB, MR, SB, RS, MM and DEB assisted in the review of the final draft for publication. All authors have read and approved the final version of this manuscript.

\section{Acknowledgements}




\section{References}

1. World Health Organization W. WHO Director-General's opening remarks at the media briefing on COVID-19 - 11 March 2020. WHO Director General's Speeches 2020:4.

2. From Wikipedia the free encyclopedia. 2020 coronavirus pandemic in Pakistan n.d.

3. Gibson L, Rush D. Novel Coronavirus in Cape Town Informal Settlements: Feasibility of using informal dwelling outlines to identify high risk areas for COVID-19 transmission from social distancing perspective. (Preprint). JMIR Public Health and Surveillance 2020;6:1-9. https://doi.org/10.2196/18844.

4. Dyer O. Covid-19: Africa records over 10000 cases as lockdowns take hold. Bmj 2020;369:m1439. https://doi.org/10.1136/bmj.m1439.

5. Bekker A, Schaaf HS, Draper HR, Kriel M, Hesseling AC. Tuberculosis disease during pregnancy and treatment outcomes in HIV-infected and uninfected women at a referral Hospital in Cape Town. PLoS ONE 2016;11. https://doi.org/10.1371/journal.pone.0164249.

6. Benali G, Ramdin T, Ballot D. An audit of mother to child HIV transmission rates and neonatal outcomes at a tertiary hospital in South Africa. BMC Research Notes 2019;12. https://doi.org/10.1186/s13104-019-4617-1.

7. Gonzalez-Perez M, Sanchez-Tarjuelo R, Shor B, Nistal-Villan E, Ochando J. The BCG Vaccine for COVID-19: First Verdict and Future Directions. Frontiers in Immunology 2021;12. https://doi.org/10.3389/fimmu.2021.632478.

8. Park SE. Epidemiology, virology, and clinical features of severe acute respiratory syndrome coronavirus-2 (SARS-CoV-2; Coronavirus Disease-19). Clinical and Experimental Pediatrics 2020;63:119-24. https://doi.org/10.3345/cep.2020.00493.

9. Adhikari SP, Meng S, Wu YJ, Mao YP, Ye RX, Wang QZ, et al. Epidemiology, causes, clinical manifestation and diagnosis, prevention and control of coronavirus disease (COVID-19) during the early outbreak period: A scoping review. Infectious Diseases of Poverty 2020;9:1-12. https://doi.org/10.1186/s40249-020-00646-x.

10. Rasmussen SA, Smulian JC, Lednicky JA, Wen TS, Jamieson DJ. Coronavirus Disease 2019 (COVID19) and Pregnancy: What obstetricians need to know. American Journal of Obstetrics and Gynecology 2020;0:2020. https://doi.org/10.1016/j.ajog.2020.02.017.

11. Lam CM, Wong SF, Leung TN, Chow KM, Yu WC, Wong TY, et al. A case-controlled study comparing clinical course and outcomes of pregnant and non-pregnant women with severe acute respiratory syndrome. BJOG: An International Journal of Obstetrics \& Gynaecology 2004;111:771-4. https://doi.org/10.1111/J.1471-0528.2004.00199.X.

12. Fan C, Lei D, Fang C, Li C, Wang M, Liu Y, et al. Perinatal Transmission of COVID-19 Associated SARSCoV-2: Should We Worry? n.d. https://doi.org/10.1093/cid/ciaa226/5809260. 
13. Wang X, Zhou Z, Zhang J, Zhu F, Tang Y, Shen X. A case of 2019 Novel Coronavirus in a pregnant woman with preterm delivery. Clinical Infectious Diseases 2020:2019-21. https://doi.org/10.1093/cid/ciaa200.

14. Dong L, Tian J, He S, Zhu C, Wang J, Liu C, et al. Possible Vertical Transmission of SARS-CoV-2 from an Infected Mother to Her Newborn. JAMA - Journal of the American Medical Association 2020:E13. https://doi.org/10.1001/jama.2020.4621.

15. Zaigham M, Andersson O. Maternal and Perinatal Outcomes with COVID-19: a systematic review of 108 pregnancies. Acta Obstetricia et Gynecologica Scandinavica 2020:0-3. https://doi.org/10.1111/aogs.13867.

16. Hong H, Wang Y, Chung HT, Chen CJ. Clinical characteristics of novel coronavirus disease 2019 (COVID-19) in newborns, infants and children. Pediatrics and Neonatology 2020. https://doi.org/10.1016/j.pedneo.2020.03.001.

17. Smith V, Seo D, Warty R, Payne O, Salih M, Chin KL, et al. Maternal and neonatal outcomes associated with COVID-19 infection: A systematic review. PLoS ONE 2020;15. https://doi.org/10.1371/journal.pone.0234187.

18. Trevisanuto D, Cavallin F, Cavicchiolo ME, Borellini M, Calgaro S, Baraldi E. Coronavirus infection in neonates: A systematic review. Archives of Disease in Childhood: Fetal and Neonatal Edition 2021;106:F330-5. https://doi.org/10.1136/archdischild-2020-319837.

19. Kimberlin DW, Stagno S. Can SARS-CoV-2 Infection Be Acquired in Utero?: More Definitive Evidence Is Needed. JAMA - Journal of the American Medical Association 2020:E1-2. https://doi.org/10.1001/jama.2020.4868.

20. Chen H, Guo J, Wang C, Luo F, Yu X, Zhang W, et al. Clinical characteristics and intrauterine vertical transmission potential of COVID-19 infection in nine pregnant women: a retrospective review of medical records. The Lancet 2020;395:809-15. https://doi.org/10.1016/S0140-6736(20)30360-3.

21. Han Y, Ma H, Suo M, Han F, Wang F, Ji J, et al. Clinical manifestation, outcomes in pregnant women with COVID-19 and the possibility of vertical transmission: a systematic review of the current data. Journal of Perinatal Medicine 2020;48:912-24. https://doi.org/10.1515/.jpm-2020-0431.

22. Schwartz DA. An Analysis of 38 Pregnant Women with COVID-19, Their Newborn Infants, and Maternal-Fetal Transmission of SARS-CoV-2: Maternal Coronavirus Infections and Pregnancy Outcomes. Archives of Pathology \& Laboratory Medicine 2020. https://doi.org/10.5858/arpa.20200901-SA.

23. Ventevogel P, Spiegel PB, Mayeya J, Dorsey S, Cohen JA, Baud D, et al. Vertical Transmission of Coronavirus Disease 19 (COVID-19) from Infected Pregnant Mothers to Neonates: A Review. The Lancet Infectious Diseases 2020;3099:30192. https://doi.org/10.1080/15513815.2020.1747120.

24. Guidance I. Infant and Young Child Feeding in the Context of the COVID-19 Pandemic Eastern , Central and Southern Africa 2020;6736:1-4.

25. Furlow B. US NICUs and donor milk banks brace for COVID-19. The Lancet Child \& Adolescent Health 2020;4642:30103. https:// doi.org/10.1016/s2352-4642(20)30103-6. 
26. Wyckoff AS. AAP issues guidance on infants born to mothers with suspected or confirmed COVID-19. AAP News 2020;2019:2019-21.

27. Stuebe A. Should Infants Be Separated from Mothers with COVID-19? First, Do No Harm. Breastfeeding Medicine 2020:bfm.2020.29153.ams. https://doi.org/10.1089/bfm.2020.29153.ams.

28. Shen K, Yang Y, Wang T, Zhao D, Jiang Y, Jin R, et al. Diagnosis, treatment, and prevention of 2019 novel coronavirus infection in children: experts' consensus statement. World Journal of Pediatrics 2020. https://doi.org/10.1007/s12519-020-00343-7.

29. Chen D, Yang H, Cao Y, Cheng W, Duan T, Fan C, et al. Expert consensus for managing pregnant women and neonates born to mothers with suspected or confirmed novel coronavirus (COVID-19) infection. International Journal of Gynecology \& Obstetrics 2020. https://doi.org/10.1002/ijgo.13146.

30. COLLECTION OF NASO/OROPHARYNGEAL SWABS FOR DETECTION OF RESPIRATORY VIRUSES. n.d.

31. Harris P a., Taylor R, Thielke R, Payne J, Gonzalez N, Conde JG. Research Electronic Data Capture (REDCap) - A metadata driven methodology and workflow process for providing translational research informatict support. Journal of Biomedical Informatics 2009;42:377-81. https://doi.org/10.1016/j.jbi.2008.08.010.Research.

32. Allotey J, Stallings E, Bonet M, Yap M, Chatterjee S, Kew T, et al. Clinical manifestations, risk factors, and maternal and perinatal outcomes of coronavirus disease 2019 in pregnancy: Living systematic review and meta-analysis. The BMJ 2020;370. https://doi.org/10.1136/bmj.m3320.

33. Sutton D, Fuchs K, D'Alton M, Goffman D. Universal Screening for SARS-CoV-2 in Women Admitted for Delivery. New England Journal of Medicine 2020;382:2163-4. https://doi.org/10.1056/nejmc2009316.

34. Villar J, Ariff S, Gunier RB, Thiruvengadam R, Rauch S, Kholin A, et al. Maternal and Neonatal Morbidity and Mortality Among Pregnant Women With and Without COVID-19 Infection. JAMA Pediatrics 2021. https://doi.org/10.1001/jamapediatrics.2021.1050.

35. Blencowe H, Calvert C, Lawn JE, Cousens S, Mathstat D, Campbell OMR. Best Practice \& Research Clinical Obstetrics and Gynaecology Measuring maternal, foetal and neonatal mortality: Challenges and solutions. Best Practice \& Research Clinical Obstetrics \& Gynaecology 2016;36:14-29. https://doi.org/10.1016/j.bpobgyn.2016.05.006.

36. Hedermann G, Hedley PL, Bækvad-Hansen M, Hjalgrim H, Rostgaard K, Poorisrisak P, et al. Danish premature birth rates during the COVID-19 lockdown. Archives of Disease in Childhood: Fetal and Neonatal Edition 2021;106:F93-5. https://doi.org/10.1136/archdischild-2020-319990.

37. No Evidence of Infectious SARS-CoV-2 in Human Milk: Analysis of a Cohort of 110 Lactationg Women n.d.

38. Norman M, Navér L, Söderling J, Ahlberg M, Hervius Askling H, Aronsson B, et al. Association of Maternal SARS-CoV-2 Infection in Pregnancy With Neonatal Outcomes. Jama 2021. https://doi.org/10.1001/jama.2021.5775. 


\section{Figures}

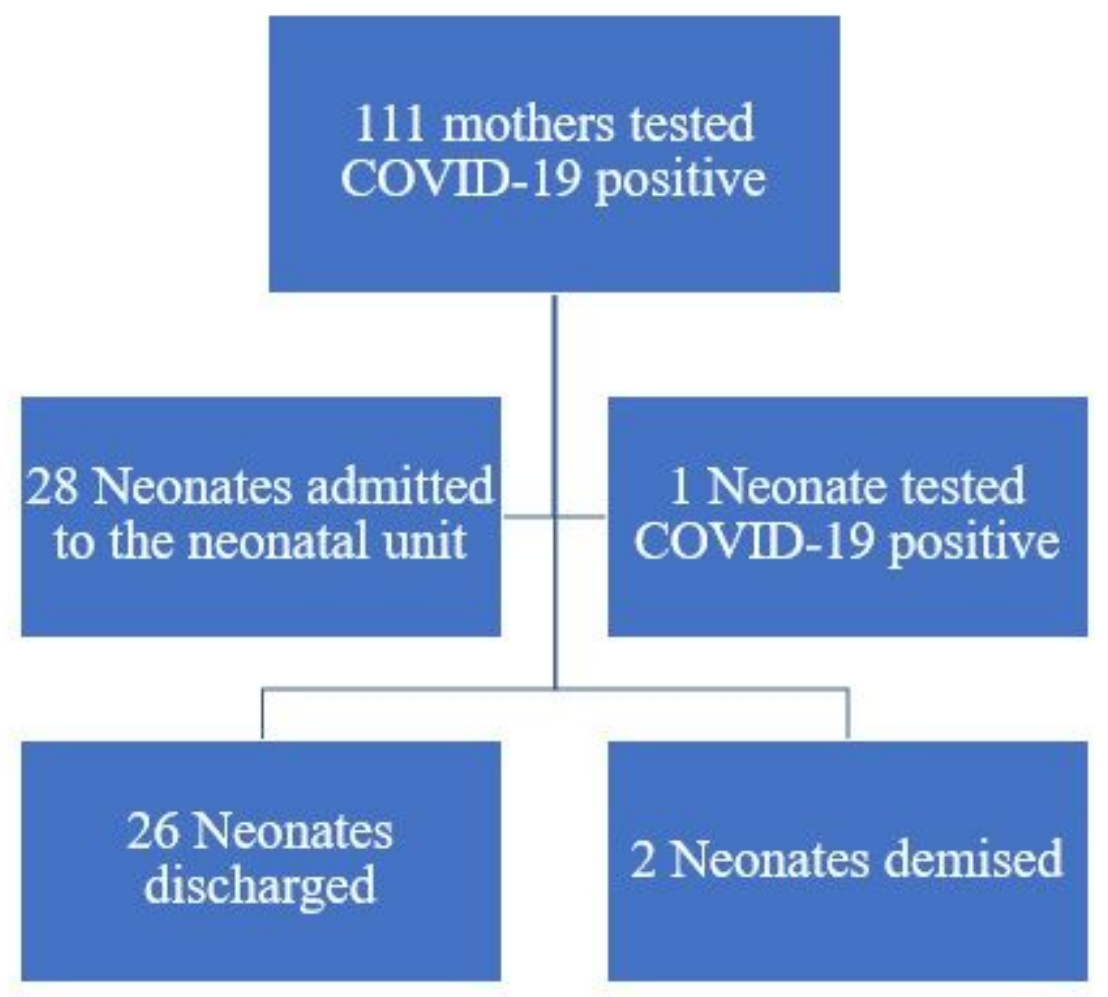

Figure 1

Sample of neonates born to COVID-19 positive mothers admitted to the neonatal unit, at a tertiary hospital in Johannesburg, South Africa, 1 August 2020 - 31 March 2021. 